\title{
Microbiological stability tests with simulated broth preparations and integrity testing for sterile standard cytotoxic preparations
}

https://doi.org/10.1515/PTHP-2020-0002

Received February 8, 2020; accepted March 30, 2020

\section{Abstract}

Objectives: The objectives were to assess the microbiological stability and the physical enclosure integrity of the overwrapping for batch production of standard cytotoxic injectable solutions.

Methods: Broth culture media were used in place of cytotoxic drugs to assess the worst case in term of microbiological contamination risk. Iterative sterility tests on batches were performed for 60 days using rapid microbiological method. Validation of microbiological growth of culture media was assessed by direct inoculation of $<100$ $\mathrm{CFU} / \mathrm{mL}$ of six microbiological strains recommended by European Pharmacopeia. Validation of the ability of growth of microorganisms in 11 cytotoxic solutions and one monoclonal antibody was assessed using eight strains. In addition, the physical integrity of the seal of the overwrapping containing cytotoxic preparations was assessed by dynamometric method and dye penetration test.

Results: No microbiological contamination was observed on all units of batches for 60 days of investigation. The ability to detect microbiological growth in cytotoxic solutions was validated for the eight challenge microorganisms after $1 / 10$ dilution for cytotoxic investigated, except for 5 Fluorouracil, gemcitabine and cisplatin. In addition, physical integrity testing of the seal of overwrapping pointed out the need of specific validation of heatsealer and operator education.

Conclusions: Besides physico-chemical testing, microbiological stability testing combined to physical integrity

\footnotetext{
*Corresponding author: Sylvie Crauste-Manciet, University of Bordeaux, ARNA Laboratory U1212 INSERM, UMR 5320 CNRS, Bordeaux, France; and University Hospital of Bordeaux (CHU), Bordeaux, France, E-mail: sylvie.crauste-manciet@chu.bordeaux.fr Agnès Matheron, Marie-Noelle Guerault, Raphael Vazquez and Mireille Cheron: CHI Poissy Saint Germain-en-Laye, Saint Germain-en-Laye, France

Denis Brossard: CHI Poissy Saint Germain-en-Laye, Saint Germain-enLaye, France; Paris Descartes University, Faculty of Pharmacy, Paris, France
}

testing is the additional part of the development method for batch production of sterile drugs in hospital.

Keywords: batch production; cytotoxic drugs; dye penetration test; microbiological stability; overwrapping; physical integrity test; rapid microbiological methods; sterility test.

\section{Introduction}

This last decade, there are increasing needs for cytotoxic drug preparation due to the increasing number of cancers treated correlated to the increasing aging of people [1]. This will become problematic issue to be afforded by hospital pharmacies where the preparation of cytotoxic drugs has to be performed due to risk of occupational exposure [2]. The development of batch production of standardized dose of cytotoxic drugs will offer interesting solution according to previous experiences of dose-banding developed in the UK [3-5]. A system where chemotherapy doses, calculated by Body Surface Area (BSA) or other means, are fitted to predefined dose ranges or "bands". For each band a standard dose (mid-point of band) is provided with standard, premade syringes or infusion bags used singly or in combination to obtain a standard dose of maximum $\pm 5 \%$ of the corresponding calculated BSA individualized dose. This concept has been proven benefits on improving workload for pharmaceutical team and nurses without measurable decrease in treatments outcomes of cancer patients $[6,7]$. Some widely used cytotoxic drugs have been previously dose-banded or dose-standardized [8-10] (i. e., carboplatin, cisplatin, paclitaxel, docetaxel, gemcitabine and monoclonal antibodies [10]). Batch production enables to improve quality control, in particular allowing microbiological control of the batch for pharmaceutical release. The issue, independently of the physicochemical stability of the drug, is the microbiological stability during storage time. To the best of our knowledge, only few studies have been studying microbiological stability [11, 12] and United States Pharmacopeia (USP) chapter 797 [13] recommends in the absence of studies, shelf-life of 9 days for medium risk level preparations or 14 days at $+4^{\circ} \mathrm{C}$ for low 
risk level preparation and for both levels 45 days when stored at $-20{ }^{\circ} \mathrm{C}$. In order to extend those durations, our objective was to determine the long-term microbiological stability for 60 days at $+4^{\circ} \mathrm{C}$ of our standard cytotoxic preparations. Parenteral preparations were performed inside ISO 5 gas sterilized isolator by aseptic transfer using filling pump. In combination to iterative sterility tests, and to exclude any false negative result due to the possible inhibition of growth by cytotoxic drugs [14], growth promotion tests with European Pharmacopeia [15] strains were performed. In addition, integrity physical tests of the sealing were performed to assess the ability of the overwrapping to maintain the enclosure integrity during the storage period.

\section{Material and methods}

\section{Material}

Microbiological strains used for microbiological assays were provided by Institut Pasteur, Paris, France for Bacillus subtilis subsp spizizenii CIP 52.62, Staphylococcus aureus subsp aureus CIP 4.83, Pseudomonas aeruginosa CIP 82.118, Clostridium sporogenes CIP 79.3, Staphylococcus epidermidis CIP 81.55, Escherichia coli CIP 54.8, Enterococcus faecalis CIP 103015, Candida albicans UMIP 48.72, and from Centre International de Ressources Microbiologiques, Marseille, France for Aspergillus niger BRFM430.

Simulated broth preparations: Pump for bag filling: Repeater®,Baxa, Bracknell, UK

Facility: isolator (Getinge Life Sciences, Tournefeuille, France) sterilized by gas process using peracetic acid (Soproper, Bioxal SA, Chalon sur Saône, France)

Detergent and disinfectant for facility cleaning: Surfa'Safe ${ }^{\circledR}$, Anios Laboratoires, Lille, France.

Growth media used were 100-mL Trypticase soya vial and 5-L prefilled bag (Dilubag®) AES Chemunex, Combourg, France.
Empty Bags: Nutripoche barriere $250 \mathrm{~mL}$ and 3L, (Sartorius Stedim Biotech SA, Aubagne, France)

Sterility test: Blood culture system Bactec ${ }^{\circledR}$ using media bottles for aerobic microorganism (Bactec Plus Aerobic/F $30 \mathrm{~mL}$ ), and anaerobic microorganism (Bactec Lytic/10 Anaerobic/ F 40 mL), Becton Dickinson, Le Pont de Claix, France.

Microorganism identification: API Gallery, api 20E, api20NE, api20STREP or Vitek-2 compact Biomerieux, Marcy l'Etoile, France.

Integrity testing of the overwrapping: Tensile strength of the sealing was measured using a DY-20B dynamometer (Adamel Lhomargy Divisions d'instruments S.A, Ivry sur seine, France) with strength cell of 100 daN.

Dye test was using a mixture of toluidine blue (Pure toluidine blue, ref 361590-0025, RAL diagnostics, Martillac, France) and Triton X-100 surfactant (Triton-X-100 solution 10\% (w/w), Volusol, VWR, Fontenay-sous-Bois, France).

Heat sealers assessed were S422VM (Heat sealer S422VM, Ora, Trevoux, France) and TISH-400 (Impulse heat sealer TISH-400, TEW, Dardilly, France)

Optical microscopy: Microscope, ref B-500TPL, Optika, Ponteranica, Italy and optika camera (Camera M699, Optika, Ponteranica, Italy) with optika vision pro software (Optika Vision Pro software, version 2.7, Optika, Ponteranica, Italy) for images capture.

Cytotoxic solutions used are given in Table 1. The diluents Dextrose solution 5\%, and Sodium chloride solution 0.9\%, (100, 250, $500 \mathrm{~mL}$ ) were provided by Macoflex, Macopharma, Mouvaux, France.

\section{Methods}

Batch production of simulated broth preparations: To assess the real batch production process, aseptic processing production with aseptic transfer was performed with the help of a peristaltic pump under aseptic conditions in an ISO 5 gas sterilized isolator but cytotoxics drugs were substituted with liquid culture medium (tryptone soya broth) [14]. The growth media used were $100-\mathrm{mL}$ vials for simulation of the drug vial and 5-L prefilled bag for simulation of diluents. The production process allowed simulating the two key

Table 1: Anticancer drug solutions investigated for batch production.

\begin{tabular}{|c|c|c|c|c|}
\hline Drug & Initial concentration, $\mathrm{mg} / \mathrm{mL}$ & Provider & diluent & Final concentration, $\mathrm{mg} / \mathrm{mL}$ \\
\hline 5 Fluorouracil & 50 & Accord $® /$ Téva $\circledR$ & D & 22 \\
\hline Gemcitabine & $40 \& 100$ & Accord $\circledast$ & NS & 10 \\
\hline Carboplatin & 10 & Accord $\circledast$ & $\mathrm{D}$ & 2 \\
\hline Cisplatin & 1 & Mylan® & NS & 0.2 \\
\hline Oxaliplatin & 5 & Accord $®$ & $\mathrm{D}$ & 0.5 \\
\hline Epirubicin & 2 & Hospira ${ }^{\circledR}$ & - & 2 \\
\hline Cyclophosphamide & 20 & Baxter ${ }^{\circledR}$ & NS & 4 \\
\hline Docetaxel & 10 & Hospira ${ }^{\circledR}$ & NS & 0.68 \\
\hline Paclitaxel & 6 & Hospira ${ }^{\circledR} /$ Teva ${ }^{\circledR}$ & NS & 0.6 \\
\hline Etoposide phosphate & 20 & Bristol-Myers Squibb $®$ & NS & 1 \\
\hline Irinotecan & 20 & $\operatorname{Accord} \circledast$ & NS & 1,15 \\
\hline Trastuzumab & 20.83 & Roche $®$ & NS & 2.25 \\
\hline
\end{tabular}

D: dextrose $5 \%$.

NS: sodium chloride $0.9 \%$. 
steps of aseptic transfer: (i) withdrawal from vials and prefilled bag and transfer of both solutions to empty big bag; (ii) withdrawal previous mixture from big bag to empty minibags. For each batch production (Figure 1), preparation of a big bag of $2,500 \mathrm{~mL}$ final volume in an empty big bag was performed by adding culture media from vials and prefilled bag, followed by the filling of $100 \mathrm{~mL}$ into 20 empty minibags. Preparation process simulating the further real process was performed in triplicate to obtain three independent batches of 20 bags, giving a total of 60 preparations performed. Final preparations were stored at $+4^{\circ} \mathrm{C}$ prior microbiological analysis. The choice of $+4^{\circ} \mathrm{C}$ was justified to reproduce the further real conditions of storage of standardized preparations. After each batch production, the isolator was cleaned and disinfected carefully with a readyto-use detergent and disinfectant followed by a gas sterilization process with peracetic acid.

Sterility test: Rapid microbiological method based on $\mathrm{CO}_{2}$ detection of

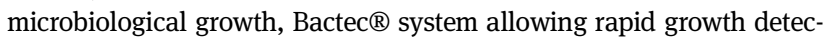
tion for bacteria within 24-48 h was chosen. For each batch, iterative sterility tests using both aerobic and anaerobic culture media bottles were performed on each of the 20 final preparations at Day 0 giving analysis of the whole batch and on five bags at Day 7, Day 14, Day 30 and Day 60 after storage at $+4^{\circ} \mathrm{C}$. Aerobic and anaerobic culture media bottles were inoculated with $10-\mathrm{mL}$ of each preparation. For iterative sterility tests, to prevent any false contamination when inoculating the culture media bottles, vacuum transfer devices and culture media bottles were individually overwrapped with each preparation in sterile container. After inoculation, the culture media bottles were transferred into a rapid microbiological detection system for a 5-day analysis. In addition, each preparation was incubated for 14 days $\left(7\right.$ days at $+25^{\circ} \mathrm{C}$ and 7 days at $+35^{\circ} \mathrm{C}$ ) according to USP $<71>[16]$ and the French Society of Pharmaceutical Sciences and Techniques (SFSTP) recommendations [17] and subjected to daily visual inspection (Figure 1), held in front of natural light to detect any visual turbidity sign of microbiological growth. This task was performed by the same investigator throughout the study to avoid variability between operators. Successful completion of the test is defined as the growth medium packaged in the plastic bag remaining a uniform, clear, light-amber solution.
Growth promotion tests: According to the recommendations of the European pharmacopeia [15], validation of the sterility test was performed by using a growth promotion test. This test was performed on all the culture media used in the study. The prefilled culture media, the culture media vials and the culture media bottles Bactec $($ ) were assessed at Day 0 and the minibags after 60 days of storage at $+4^{\circ} \mathrm{C}$ (end of study period) to demonstrate media growth performances. The six following strains recommended by European Pharmacopeia [15] were investigated including: two aerobic Gram-positive bacteria, (B. subtilis subsp spizizenii, S. aureus subsp aureus), one aerobic Gram-negative bacterium ( $P$. aeruginosa), one anaerobic Gram-positive bacterium, (C. sporogenes) one fungus (A. niger) and one yeast (C. albicans). Strains were harvested by using Brain-Heart Infusion broth (BHI) and serially diluted to achieve a final concentration $<100$ Colony Forming Units (CFU)/mL. Colony counts were carried out to confirm concentrations by sprawling $1 \mathrm{~mL}$ of each inoculum on specified agar. All the strains were prepared by the same experienced laboratory technician throughout the study to avoid operator-dependent variability. The growth promotion test was carried out aseptically by adding into each unit $1 \mathrm{~mL}$ of BHI broth containing $<100 \mathrm{CFU} / \mathrm{mL}$ of the appropriate bacterium, fungus or yeast. When microbiological growth was visually detected and to exclude any cross contamination of environmental strains, microorganisms were identified by the microbiology department of the hospital using a Vitek-2 microbiological identification system or API gallery and only a direct examination for $A$. niger. Some bags and vials of culture medium not subjected to inoculation were incubated simultaneously to the inoculated bags and vials as negative controls.

Integrity testing of the overwrapping: We considered the primary containers of preparations empty bag of Ethylene Vinyl Acetate/ Ethylene Vinyl Alcohol Copolymer (EVA/EVOH) were safe for maintenance of integrity and sterility. Thus, the critical point to be assessed would be the overwrapping and in particular the sealing which is manually performed. Overwrapping of preparation is individually performed on each preparation by sealing a disposable sterile Biosafe containers (90 $\mu \mathrm{m}$-thick sterile polyethylene tubing Biosafe bio 103) which is leak tight connected to interlocking door fitted with isolators'

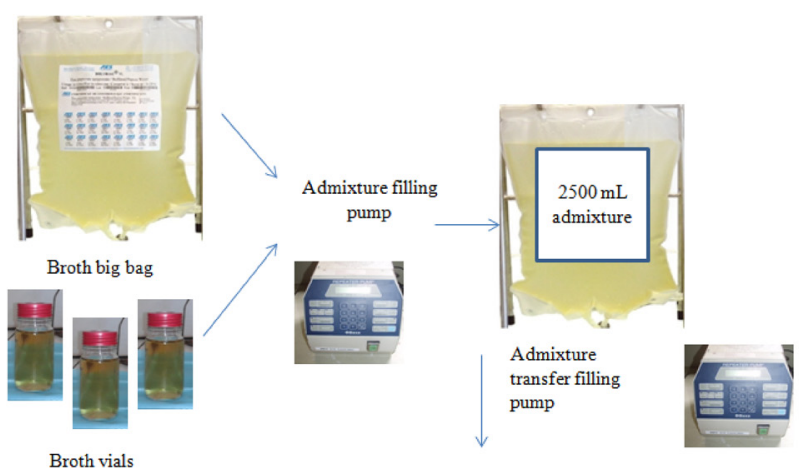

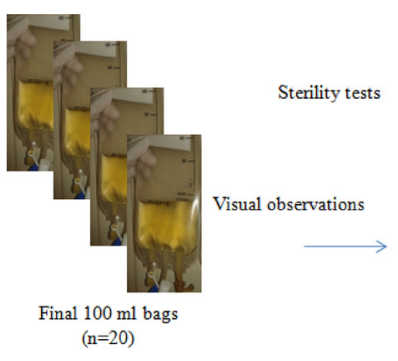

$(\mathrm{n}=20)$

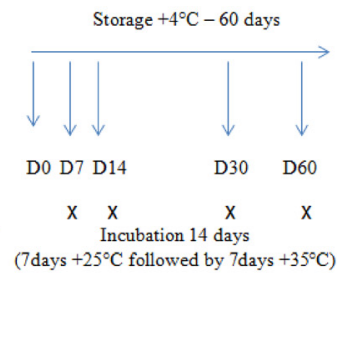

Figure 1: Process simulation method and sterility analysis per batch. 
wall. This system enables to ensure simultaneously the overwrapping of final product and the containment of the sterile content.

Two physical evaluations were performed on the sealing of the overwrapping: (i) the measure of tensile strength giving a rupture of the sealing using a DY-20B dynamometer and (ii) the dye penetration test which allows the visual and microscopic detection of leaks of the sealing using a mixture of toluidine blue and Triton X-100 surfactant according to standard ASTM method [18]. Both visual and microscopic observations of the seals were performed using two heat sealers S422VM and TISH-400 giving a $2 \mathrm{~mm}$ wide seal. The differences in the integrity between S422VM sealer and TISH-400 sealer were statistically analysed by Student test and ANOVA test.

For both heat sealers, the best sealing conditions (time and temperature) was previously fixed using dynamometric measurements and fixed to be comparable to dynamometric resistance of the lateral seals of commercialized polypropylene empty bag (i. e., multilayer bags SLB medical, Genas, France)

Impact of operators on the sealing quality of the seal was assessed for both heat sealers using dye penetration test. The Fisher exact test was used to estimate the statistical differences in dye test between the two heat sealers and between experienced and nonexperienced operators.

For the best heat sealer, impact of temperature on the seal quality (i. e. $+4^{\circ} \mathrm{C}$ and $\left.-22^{\circ} \mathrm{C}\right)$ in comparison to ambient temperature $\left(+22^{\circ} \mathrm{C}\right)$ after 60 days of storage was investigated both by dynamometric measurement and dye penetration test. Dynamometer measurements and dye test results were statistically analysed by Fisher exact test.

\section{Validation of microbiological growth with cytotoxic drugs}

The ability of sterility test using Bactec ${ }^{\circledR}$ system to be relevant to detect microbiological growth on real batches of cytotoxic drugs was assessed by additional microbiological growth promotion test with cytotoxic bags. Eleven cytotoxic drugs and one monoclonal antibody at final concentration usually used for clinical administration (Table 1) were investigated for growth promotion test. Five microorganisms recommended by European Pharmacopeia [15] B. subtilis, Staphylococcus aureus, $P$. aeruginosa, A. niger and $C$. albicans and three environmental strains. (Staphylococcus epidermidis, E. coli and E. faecalis) which could be responsible of environmental contamination coming from human contamination [19] were directly inoculated in cytotoxic bag at a final concentration $<100 \mathrm{CFU} /$ unit. Inoculation was performed outside controlled areas but with respect of aseptic conditions. Ten milliliter of each cytotoxic bag challenged with microorganism was transferred with a vacutainer ${ }^{\circledR}$ system in an appropriate Bactec ${ }^{\circledR}$ aerobic or anaerobic. Solvent solutions without cytotoxic drugs (sodium chloride solution $0.9 \%$ and dextrose solution $5 \%$ ) were used as positive control to show the microbiological growth. When no microbiological growth was detected, 1/10 dilution of the initial cytotoxic solutions was reassessed on Bactec $®$ system.

\section{Results}

\section{Long term stability assessment}

\section{Sterility test}

For the three batches produced, results of iterative sterility tests performed with rapid microbiological method (Bactec $₫$ system) were negative at Day 0 ( 60 bags) and at Day 7 (15 bags), Day 14 (15 bags) , Day 30 (15 bags) and Day 60 (15 bags) (Table 2).

None of the 60 preparations incubated at $+25{ }^{\circ} \mathrm{C}$ for 7 days and then at $+35^{\circ} \mathrm{C}$ for seven 7 exhibited turbidity at the end of the 14-day visual observation period (Table 3).

Table 2: Results of sterility tests of bags batch produced at Day 0, Day 7, Day 14, Day 30 and Day 60 using rapid microbiological method (Bactec () .

\begin{tabular}{lllllll}
\hline Sterility test $n^{\circ}$ & Bactec $@$ media & $\begin{array}{l}\text { Day } 0 \\
(n=60 \text { bags })\end{array}$ & $\begin{array}{l}\text { Day } 7 \\
(n=15 \text { bags })\end{array}$ & $\begin{array}{l}\text { Day14 } \\
(\mathrm{n}=15 \text { bags })\end{array}$ & $\begin{array}{l}\text { Day30 } \\
(\mathrm{n}=15 \text { bags })\end{array}$ & $\begin{array}{l}\text { Day 60 } \\
(\mathrm{n}=15 \text { bags })\end{array}$ \\
\hline 1 & aerobic & - & - & - & - & - \\
& anaerobic & - & - & - & - & - \\
2 & aerobic & - & - & - & - & - \\
3 & anaerobic & - & - & - & - & - \\
& aerobic & - & - & - & - & - \\
\hline
\end{tabular}

- : lack of growth.

Table 3: Results of visual inspection of bags batch produced after incubation at $+25^{\circ} \mathrm{C}$ for 7 days followed by incubation at $+35^{\circ} \mathrm{C}$ for 7 days.

\begin{tabular}{lllll}
\hline & $\begin{array}{l}\text { Day } 7 \\
\text { (n=15 bags) }\end{array}$ & $\begin{array}{l}\text { Day } 14 \\
(\mathrm{n}=15 \text { bags })\end{array}$ & $\begin{array}{l}\text { Day } 30 \\
(\mathrm{n}=15 \text { bags })\end{array}$ & $\begin{array}{l}\text { Day 60 } \\
(\mathrm{n}=15 \text { bags })\end{array}$ \\
\hline Sterility test 1 & - & - & - & - \\
Sterility test 2 & - & - & - & - \\
Sterility test 3 & - & - & - & - \\
\hline
\end{tabular}

(-) lack of turbidity. 


\section{Growth promotion test}

The eight challenge microorganisms inoculated at a concentration $<100 \mathrm{CFU}$ were found to grow inside all culture media used in the study i. e. culture media bags and vials, culture media bags batch produced and Bactec ${ }^{\circledR}$ culture media. Due to anaerobic metabolism of C. sporogenes, growth promotion was only possible on Bactec ${ }^{\circledR}$ anaerobic culture media. Moreover, the preservation of fertility of the culture media batch produced was confirmed after 60 days of storage at $+4^{\circ} \mathrm{C}$ with the eight challenge microorganisms.

Table 4: Results of toluidine blue dye penetration tests with regards to heat sealer (S422 VM, TISH-400) and operators (experienced and inexperienced).

\begin{tabular}{lrr}
\hline & S422 VM & TISH-400 \\
\hline Total number of seals & 80 & 130 \\
Number of operators & 8 & 11 \\
Number of seals function & & \\
$\quad$ of operators skill & & \\
experienced & 60 & 72 \\
inexperienced & 20 & 58 \\
Total correct seals & $71 / 80$ & $128 / 130$ \\
$\quad$ [number/total] & & \\
$\%$ & 88,75 & 98,46 \\
Experienced [number/total] & $55 / 60$ & $72 / 72$ \\
$\%$ & 91,7 & 100 \\
Inexperienced [number/total] & $16 / 20$ & $56 / 58$ \\
$\%$ & 80 & 96,6 \\
Total defect seals & $9 / 80$ & $2 / 130$ \\
$\quad$ [number/total] & & \\
$\%$ & 11,25 & 1,54 \\
Experienced [number/total] & $5 / 60$ & $0 / 72$ \\
$\%$ & 8.3 & 0 \\
Inexperienced [number/total] & $4 / 20$ & $2 / 58$ \\
$\%$ & 20 & 3,45 \\
\hline
\end{tabular}

\section{Integrity testing of the overwrapping of cytotoxic bags}

Dynamometric results of five measurements of the resistance of seals using optimized parameters for heat sealing were $7.74 \pm 0.07 \mathrm{daN}$ and $7.69 \pm 0.27 \mathrm{daN}$ for S422VM and TISH-400 respectively. These results were not significantly different but were significantly different $(p<0.00001$, ANOVA test) compared to dynamometric results obtained for the resistance of lateral seal of commercialized polypropylene empty bag SLB which were giving $9.19 \pm 0.19$ daN. However, the rupture resistance of lateral seal of the multilayer bag used in our study was significantly much higher $\left(\mathrm{p}<10^{-5}\right.$, ANOVA test) giving $26.06 \pm 1.6 \mathrm{daN}$.

With these optimized conditions, significant differences ( $p<0.01$, Fisher exact test, $\alpha=0.05 \%$ ) were found between the two heat sealers in favour of the TISH- 400 where less defects were found after dye penetration test (Table 4). No significant difference was observed between inexperienced and experienced operators for S422VM and TISH400 (Table 4).

With the best heat sealer giving significantly less defects TISH-400, we noticed a significant decrease in the resistance $(p<0.0001$, ANOVA test) of the sealing when stored 60 days at cold and freezing temperatures giving $6.43 \pm 0.22 \mathrm{daN}$ and $7.01 \pm 0.31$ daN for storage at $+4{ }^{\circ} \mathrm{C}$ and $-22{ }^{\circ} \mathrm{C}$ respectively compared to $7.77 \pm 0,32$ when stored 60 days at $+22^{\circ} \mathrm{C}$. However, there were no significant differences of number of defect seals investigated by dye penetration test in comparison with seals stored at $+22^{\circ} \mathrm{C}$. Over the 66 samples stored at $+4{ }^{\circ} \mathrm{C}$ and $-22^{\circ} \mathrm{C}$, only one defect seals was noticed after storage at $+4^{\circ} \mathrm{C}$ and no defect seal after storage at $-22^{\circ} \mathrm{C}$.

Two major defects were identified: the presence of channels within the seal and the lack of seal on the edge of the overwrapping. Visual observations of those defects are given in Figure 2. Microscopic observations with blue dye

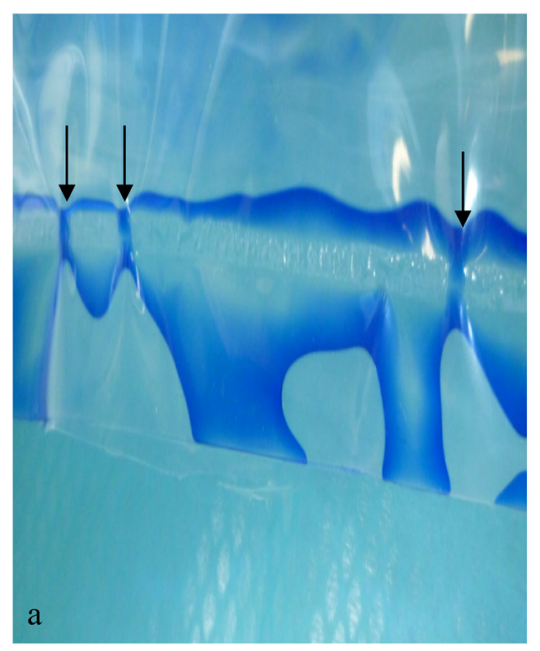

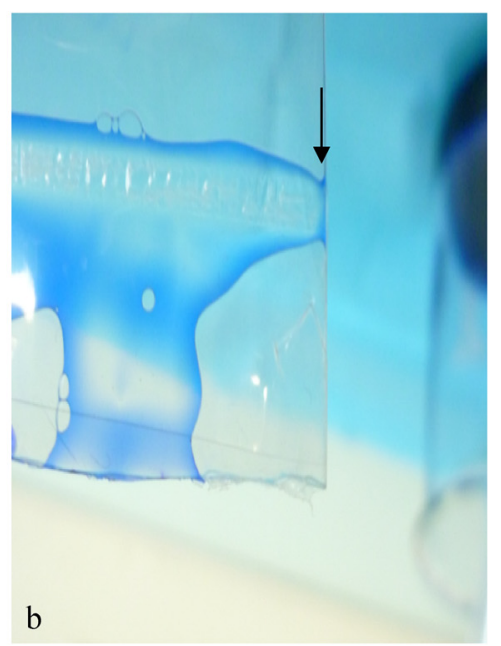

Figure 2: Visual observation of defect seals when using S422VM heat sealer. Dye solution introduced inside the overwrapping allowed to visually detecting leaks within the seal. Example of leaks due to incomplete sealing are identified on the pictures by black arrows (a) multiple leaks throughout the sealing (b) single leak due to incomplete sealing of the overwrapping edge. 

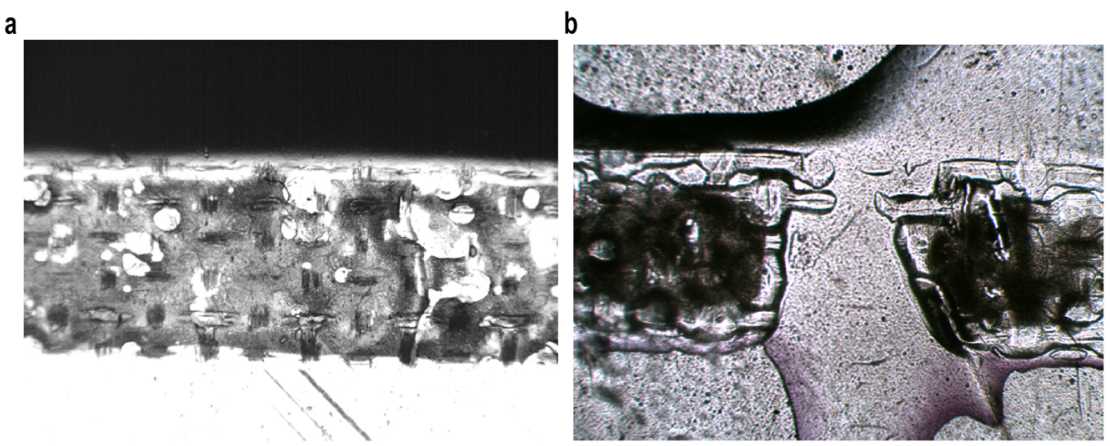

Figure 3: Microscopic observations (magnification $\times 20$ ) of correct seals and defect seals using toluidine blue dye penetration test with S422VM heat sealer. (a) correct seal. (b) defect seal. of correct and defect seals using the S422VM sealer are given in Figure 3.

\section{Validation of the microbiological growth with cytotoxic drug solutions in Bactec $\circledast$ culture media}

Results of the growth of the eight challenge microorganisms are given in Table 5 . The growth of the eight microorganisms was possible only with undiluted solutions of cyclophosphamide and trastuzumab. For the remaining cytotoxic solutions, in most of the case undiluted cytotoxic solutions were able to inhibit growth of $P$. aeruginosa, B. subtilis and E. coli.
Except for 5 Fluorouracil, gemcitabine and cisplatin, 1/10 dilution of the cytotoxic solutions was enough to recover the growth for the eight microorganisms investigated. For 5 Fluorouracil, 1/10 dilution allowed to recover $C$. albicans and E. coli, but none of the other species. For gemcitabine, 1/10 dilution allowed the recovering of growth of $E$. coli, but $1 / 10$ dilution was not able to recover the growth of Staphylococcus species, $B$. subtilis and E. faecalis. For cisplatin, 1/10 dilution allowed the recovering of $B$. subtilis and $E$. coli, but not $P$. aeruginosa. When microbiological growth was observed, the result given by the automated system was always obtained within $48 \mathrm{~h}$.

Table 5: Results of microbiological growth of eight species using rapid microbiological method with concentrated and diluted cytotoxic drugs solutions.

\begin{tabular}{|c|c|c|c|c|c|c|c|c|c|c|}
\hline Drug & Method & $\mathrm{C}^{\circ}, \mathrm{mg} / \mathrm{mL}$ & SA & PA & BS & CA & AN & SE & $\mathrm{EC}$ & EF \\
\hline \multirow[t]{2}{*}{5 Fluorouracil } & Bactec $®$ & 22 & - & - & - & - & - & - & - & - \\
\hline & & 2.2 & - & - & - & + & - & - & + & - \\
\hline \multirow[t]{2}{*}{ Gemcitabine } & Bactec $®$ & 10 & - & + & - & + & + & - & - & - \\
\hline & & 1 & - & + & - & + & + & - & + & - \\
\hline \multirow[t]{2}{*}{ Carboplatin } & Bactec $®$ & 2 & + & + & + & + & + & + & - & + \\
\hline & & 0.2 & & & & & & & + & \\
\hline \multirow[t]{2}{*}{ Cisplatin } & Bactec $®$ & 0.2 & + & - & - & + & + & + & - & + \\
\hline & & 0.02 & & - & + & & & & + & \\
\hline \multirow[t]{2}{*}{ Oxaliplatin } & Bactec $®$ & 0.5 & + & - & + & + & + & + & + & + \\
\hline & & 0.05 & & + & & & & & & \\
\hline \multirow[t]{2}{*}{ Epirubicin } & Bactec $®$ & 2 & - & - & - & + & + & - & + & + \\
\hline & & 0.2 & + & + & + & & & + & & \\
\hline Cyclophosphamide & Bactec $®$ & 4 & + & + & + & + & + & + & + & + \\
\hline \multirow[t]{2}{*}{ Docetaxel } & Bactec $®$ & 0.68 & + & - & - & + & + & + & + & + \\
\hline & & 0.068 & & + & + & & & & & \\
\hline \multirow[t]{2}{*}{ Paclitaxel } & Bactec $®$ & 0.6 & + & - & - & + & + & + & + & + \\
\hline & & 0.06 & & + & + & & & & & \\
\hline \multirow[t]{2}{*}{ Etoposide phosphate } & Bactec $®$ & 1 & + & - & + & + & + & + & + & + \\
\hline & & 0.1 & & + & & & & & & \\
\hline \multirow[t]{2}{*}{ Irinotecan } & Bactec $®$ & 1,15 & + & - & + & + & + & + & + & + \\
\hline & & 0.115 & & + & & & & & & \\
\hline Trastuzumab & Bactec $®$ & 2.25 & + & + & + & + & + & + & + & + \\
\hline
\end{tabular}

SA: Staphylococcus aureus - PA: Pseudomonas aeruginosa - BS : Bacillus subtilis - CA : Candida albicans- AN : Aspergillus niger - SE : Staphylococcus epidermidis - EC : Escherichia coli - EF : Enterococcus faecalis.

-: no growth after 5 days. +: growth within $48 \mathrm{~h}$. 


\section{Discussion}

To the best of our knowledge, only few studies investigated long term microbiological stabilities for hospital pharmacy preparations [11, 12] and assessing the maintenance of sterility using growth-promoting broth. In the absence of sterility testing in accordance to USP $<71>[16]$, USP $<797>$ [13] recommended shelf-lives of 14 days at $+4^{\circ} \mathrm{C}$ and $48 \mathrm{~h}$ at room temperature or 9 days at $+4^{\circ} \mathrm{C}$ and $30 \mathrm{~h}$ at room temperature for low microbiological risk and medium microbiological risk preparations respectively. The process used in our study should be considered as medium microbiological risk process in accordance to USP <797> [13], as far multiple transfers are needed to produce batch of several bags. Our study showed the possibility to extend shelf-life for 60 days in multilayer plastic bags contained in a sterile overwrapping.

For sterility testing, we used alternative method to the European Pharmacopeia standard which is membrane filtration. This method gives results after 14 days of incubation which represents about $23 \%$ loss of the period of availability of the product. This loss is reduced up to $3 \%$ when using rapid microbiological methods which give microbiological result within $48 \mathrm{~h}$. Rapid microbiological methods for sterility control of sterile preparation by adenosine triphosphate (ATP) bioluminescence technology or $\mathrm{CO}_{2}$ monitoring technologies (BacT/Alert and the BACTEC systems), were already validated for sterility testing in industry according to technical report $n^{\circ} 33$ of the Parenteral Drug Association [20] and hospital pharmacies [21, 22]. Our choice between rapid methods was dictated by the availability of Bactec $\AA^{\circledR}$ system for blood culture in our hospital. The method allowed nondestructive assay and close transfer of the solution into the culture medium avoiding false positive results. In addition, the closed transfer and the containment in a leakproof culture medium bottle allow avoiding spreading off chemical contamination coming from the cytotoxic solutions which is recommended for limiting occupational exposure [23].

The use of culture medium instead of cytotoxic drugs was motivated both to enhance the sensibility of sterility testing giving a worst case condition and to avoid any possible risk of growth inhibition with cytotoxic drugs [24]. Simulation with culture medium allowed enhancing sensibility of the microbiological assessment when producing aseptic compounds. Trissel et al. [25], showed for medium aseptic compounding using media fill tests an overall contamination rate of $5.2 \%$, whereas the risk estimated in real production conditions were much lower, with 1.9 and $0 \%$ for individualized doses preparation and batch preparation in pharmaceutical environment respectively [26]. Moreover, in pharmaceutical dedicated units, it was estimated to be lower than $2.2 \mathrm{ppm}$ by Tisdwell [27]. In our study, $100 \%$ of the units were assessed for sterility testing throughout the storage period. However, in routine, sterility test will only be a statistical evaluation of the batch which enforced the interest to combine sterility tests with physical assessment of the enclosure integrity as suggested by FDA guidance for industry [28] for container and closure system integrity testing. In our study, the first physical barrier was the bag itself, chosen to be a multilayer plastic bag not permeable to gas and oxygen. In comparison to standard polypropylene empty bag, the resistance of the lateral seal was 2.8 higher with the multilayer plastic bag used in our study. This means the primary content, which is the first barrier against microbiological contamination, offers a highest level of security to maintain sterility of the solution during the storage period. That is why in our study we did not consider the risk of microbiological contamination related to the potential ingress of microorganisms inside the primary bag. When the risk of microbiological ingress inside the primary container may be suspected i. e. with syringes, physical and microbiological assessments should be additionally performed [29]. Another limitation of our study may be that we did not assess the direct impact of the drugs on the primary container and finally impact on microbiological stability. When drug-content interactions with the primary container are suspected it is recommended to combine an additional specific evaluation with the drug according to the last GERPAC European guidance on microbiological stability [29]. The second barrier is the overwrapping of the bag able to contain the bag inside its original sterile environment. We detected that sealing of the overwrapping was the critical point for enclosure integrity. The validation of the sealing with dye test allowed us to detect the major importance of this assessment showing significant difference between two brands of heat sealers. Despite the lack of significant difference between experienced and inexperienced operators found in our study, their education to perform correct seal should be included in the education program for aseptic compounding. Operator's education was already highlighted for aseptic compounding [30] and our work pointed out the needs to investigate the whole process and not only the aseptic process.

For routine evaluation of sterility of our batch produced, we needed to check that the rapid microbiological method was able to detect the growth of microorganisms which may contaminate cytotoxic solutions. Some controversies arose from literature about microbicidal or microbiostatic activity of antitumor agents [24, 31-34], but some studies showed the 
possible growth inside cytotoxic solutions [14, 35-37] justifying our assessment. We choose challenge microorganism recommended by European pharmacopeia [15], but also we choose to investigate the growth of additional microorganisms coming from human contamination, as a potential source of contamination in clean rooms [19]. According to our results, when using Bactec $\circledast$ culture media, most of the challenged microorganisms were able to grow with concentrated cytotoxic solution except for gemcitabine and 5 Fluorouracil. The inhibitory effect of 5 Fluorouracil and gemcitabine on growth was previously described in literature $[32,33,35,38]$. Among literature, it is possible to find opposite results on inhibitory effect of anticancer drugs. For example, etoposide is able to either inhibit or promote microbiological growth [39]. Bactec $\AA$ culture media was improved for minimizing the potential microbicidal or microbiostatic activity of drugs by including resins able to bind the drug [40]. However, in our study, several challenge microorganisms were unable to grow (i. e., $P$. aeruginos $a$ ) in cytotoxic concentrated solutions, but in most of the cases, the $1 / 10$ dilution of the initial cytotoxic solution investigated was able to allow the growth of all the challenge microorganisms. Dilution of the drug solution is a usual method to overcome inhibitory effect on growth thanks to European Pharmacopeia [15]. In a similar way, it was shown for blood culture of patient treated with cytotoxic drugs that the dilution was able to restore growth of microorganisms [40]. Nevertheless, 1/10 dilution was ineffective to recover growth of Staphylococcus species, $P$. aeruginosa, B. subtilis, A. niger and $E$. faecalis in 5 Fluorouracil. It was also ineffective to recover growth in gemcitabine of staphylococcus species, Bacillus subtilis and E. faecalis. It was not possible to predict the growth inhibition in relation to the chemical structure of the cytotoxic drug, similar growth profiles of the microorganism were found with paclitaxel and docetaxel but the three platinum derived drugs showed different growth profiles. This is consistent with previous observation from Krämer et al. [41], showing the lack of link between chemical structure or pharmacological properties and their microbicidal or microbiostatic activity. Moreover, drug formulation may play important role in microbiological growth [42, 43] and even most of the pharmaceutical aqueous solutions do not promote microbiological growth, microorganism may remain viable. For routine evaluation, a 1/10 dilution of initial cytotoxic solution should be systematically applied to limit risk of false negative. Nevertheless, as previously published for 5 Fluorouracil $[33,38]$, and evidenced in our study for gemcitabine, microbiological growth of some strains could be difficult. Thus it is justifying to complete microbiological investigation with endotoxin control [44] able to evidence Gram-negative bacteria [45, 46]. Moreover, the microbiological environmental quality results of the ISO 5 compounding area pre and post-production should be in conformity with USP <797> [13] or PICS guidelines [47] to allow the batch release.

\section{Conclusion}

Our study was able to show the sterility maintenance of broth preparations simulating the batch production of cytotoxic drugs during 60 days at $+4^{\circ} \mathrm{C}$. It highlighted the interest to work on the overwrapping maintenance to maintain the initial sterile status of the preparation. Education of operators, as it is well described in USP <797> for aseptic compounding, in particular using media fill test, should be extended to related activities involved in the process and able to compromise the final sterility. Routine evaluation of the sterility could suffer from some microbiological inhibitory effects of the cytotoxic drug solutions. For batch release, it is necessary to combine the results of sterility testing, endotoxin determination and microbiological environmental results in conformity with ISO 5 environment, in accordance with international guidelines. For routine, taking into account that sterility test could be ineffective to detect some microbiological species with few cytotoxic drugs, additional investigations such as endotoxin determination and parametric release including microbiological environmental contamination results should be implemented.

Research funding: none.

Author contributions: A.M. carried out the experiments, analyzed the data, and wrote the manuscript M-N.G., R.V., M.C., D.B. and S.C-M. designed the experiments, analyzed the data, and wrote the manuscript.

Competing interests: The authors declare no competing financial interest.

\section{References}

1. Hoppe-Tichy T. Current challenges in European oncology pharmacy practice. J Oncol Pharm Pract 2010;16:9-18.

2. Connor TH, DeBord DG, Pretty JR, Oliver MS, Roth TS, Lees PS, et al. Evaluation of antineoplastic drug exposure of health care workers at three university-based US cancer centers. J Occup Environ Med 2010;52:1019-27.

3. Plumridge RJ, Sewell GJ. Dose-banding of cytotoxic drugs: a new concept in cancer chemotherapy. Am J Health Syst Pharm 2001; 58:1760-4.

4. Kaestner SA, Sewell GJ. Chemotherapy dosing part I: scientific basis for current practice and use of body surface area. Clin Oncol (R Coll Radiol) 2007;19:23-37. 
5. Kaestner SA, Sewell GJ. Chemotherapy dosing part II: alternative approaches and future prospects. Clin Oncol (R Coll Radiol). 2007;19:99-107.

6. Kaestner SA, Sewell GJ. A national survey investigating UK prescribers' opinions on chemotherapy dosing and 'dosebanding'. Clin Oncol (R Coll Radiol) 2009;21:320-8.

7. Baker JP, Jones SE. Rationalisation of chemotherapy services in the university hospital birmingham national health science trust. J Oncol Pharm Pract 1998;4:10-4.

8. Kaestner S, Sewell GJ. Dose-banding of carboplatin: rationale and proposed banding scheme. J Oncol Pharm Pract 2007;13:109-17.

9. Pouliquen AL, Escalup L, Jourdan N, Cottu P, Faure P, MadelaineChambrin I. Dose standardisation of anticancer drugs. Int J Clin Pharm 2011;33:221-8.

10. Mathijssen RH, De Jong FA, Loos WJ, Van Der Bol JM, Verweij J, Sparreboom A. Flat-fixed dosing versus body surface area-based dosing of anticancer drugs in adults: does it make a difference? Oncol 2007;12:913-23.

11. Gonzalez S, Miller D, Murphy SP. Maintenance of sterility in 1-mL polypropylene syringes. Am J Health Syst Pharm 2007;64:1962-4.

12. Tribble DA. Long-term maintenance of sterility in single-use syringes. Am J Health-Syst Pharm 2006;63:702-7.

13. Pharmaceutical Compounding - sterile preparations (general information 797). In: The United States pharmacopeia, 42nd rev., and the National Formulary, 35th ed. Rockville, MD: The United States Pharmacopeia Convention; 2019: 1-37 pp.

14. Hammilton-Miller JMT. Antimicrobiological activity of 21 antineoplastic agents. Br J Canc. 1984;49:367-9.

15. Biological methods (Chapter 2.6.1 Sterility). In: European Pharmacopeia, 7th rev. EDQM; 2013. Available from: www.edqm. eu [Accessed 5 May 2013].

16. Sterility Tests (general information 71). In: The United States pharmacopeia, 36th rev., and the national formulary, 31st ed. Rockville, MD: United States Pharmacopeial Convention; 2013.

17. Barillet L, Bechaud P, Chancel O, Faion M, Moukaabari H. De la pertinence des milieux de culture dans les simulations de procédés aseptiques. STP Pharma Prat 2008;18:503-11.

18. ASTM F1929. Standard test method for detecting seal leaks in porous medical packaging by Dye Penetration. West Conshohocken, PA, USA: ASTM International; 2012.

19. Sandle T. A review of cleanroom microflora: types, trends, and patterns. PDA J Pharm Sci Technol 2011;65:392-403.

20. Evaluation, validation and implementation of new microbiological testing methods. PDA technical report no. 33. Bethesda, MD: Parenteral Drug Association, 2000.

21. Steinmetz P, Demontrond D. Sterility controls of parenteral nutrition mixtures: «Bactec assessment». Nutr Clin Metabol 1992;6:93-7.

22. Pigué C, Jeannot JL, Jumas-Bilak E, Florent M. Contrôle microbiologique des mélanges de nutrition parentérale « à la carte » : comparaison d'un milieu diphasique pour hémoculture, de l'automate Bact/Alert ${ }^{\circledR}$ et de l'essai de stérilité selon la pharmacopée européenne. Nutr Clin Metab 2007;21:40-1.

23. National Institute for Occupational Safety and Health. NIOSH alert: Preventing occupational exposure to antineoplastic and other hazardous drugs in healthcare settings, 2004. Available from: www.cdc.gov/niosh/docs/2004-165/pdfs/2004-165.pdf [Accessed 19 May 2013].
24. Patel K, Craig SB, McBride MG, Palepu NR. Microbial inhibitory properties and stability of topotecan hydrochloride injection. Am J Health Syst Pharm 1998;55:1584-7.

25. Trissel LA, Gentempo JA, Anderson RW, Lajuenesse JD. Using a medium-fill simulation to evaluate the microbial contamination rate for USP medium-risk-level compounding. Am J Health Syst Pharm 2005;62:285-8.

26. Austin PD, Elia M. A systematic review and meta-analysis of the risk of microbial contamination of aseptically prepared doses in different environments. J Pharm Pharmaceut Sci 2009;12:23342.

27. Tidswell EC, Rockwell J, Wright MO. Reducing hospital-acquired infection by quantitative risk modeling of intravenous bag preparation. PDA J Pharm Sci Technol 2010;64:82-91.

28. US Food and Drug Administration. Guidance for industry - Container and closure system integrity testing in lieu of sterility testing as component of the stability protocol for sterile products, 2008. Available from: www.fda.gov/downloads/Regulatorylnformation/ Guidances/UCM146076.pdf [Accessed 19 May 2013].

29. Crauste-Manciet S., Krämer I., Lagarce F., Sautou V., Beaney A., Smith J, et al. GERPAC Consensus Conference - Guidance on the assignment of microbiological shelf-life for hospital pharmacy aseptic preparations. PTHP 2020, in press. https://doi.org/10. 1515/pthp-2020-0001.

30. Sigward E, Fourgeaud M, Vazquez R, Guerrault-Moro MN, Brossard D, Crauste-Manciet S. Aseptic simulation test challenged with microorganisms for validation of pharmacy operators. Am J Health Syst Pharm 2012;69:1218-24.

31. Metcalfe D, Hugues WT. Effects of methotrexate on group A beta hemolytic streptococci and streptococcal infection. Cancer 1972; 30:588-93.

32. Bodet CA, Jorgensen JH, Drutz DJ. Antibacterial activities of antineoplastic agents. Antimicrob Agents Chemother 1985;28: 437-9.

33. Holmes CJ, Kubey WY, Love DI. Viability of microorganisms in fluorouracil and cisplatin small-volume injections. Am J Hosp Pharm 1988;45:1089-91.

34. Favier B, Latour JF, Fuhrmann C. Viability of microorganisms in bags of cytotoxic drugs. J Oncol Pharm Pract. 2001;6:167-71.

35. Krämer I. Viability of microorganisms in novel antineoplastic and antiviral drug solutions. J Oncol Pharm Pract 1998;4:32-7.

36. Paris I, Paci A, Rey JB, Bourget P. Microbial growth tests in antineoplastic injectable solutions. J Oncol Pharm Pract 2005;11: $7-12$.

37. Karstens A, Krämer I. Viability of micro-organisms in novel anticancer drug solutions. Eur J Hosp Pharm Sci 2007;13:27-32.

38. Krämer I, Wenchel HM. Wachstumsverhalten ausgewählter mikroorganismen in zytostatika-zubereitungen.

Krankenhauspharmazie 1988;11:439-42.

39. Calame W, Van der Waals R, Douwes-Idema N, Mattie H, van Furth R. Antibacterial effect of etoposide in vitro. Antimicrob Agents Chemother 1988;32:1456-7.

40. Hopfer RL, Moore DG, Fainstein V, Watkins S, Wenglar M. Routine use of BACTEC 16B bottles to remove antibacterial and antitumor agents from blood cultures of cancer patients. J Clin Microbiol 1983;18:759-64.

41. Krämer I, Wenchel HM. Viability of microorganisms in antineoplastic drug solutions. Eur J Hosp Pharm 1991;1:14-9. 
42. Sarakbi I, Federici, M, Krämer, I. Viability of microorganisms in novel chemical and biopharmaceutical anticancer drug solutions. Eur J Parenter Pharm Sci 2015;20:5-12.

43. Sarakbi I, Heeb R, Thiesen J, Krämer I. Viability of selected microorganisms in non-cytotoxic aseptic preparations.

Pharmaceut Technol Hosp Pharm 2016;1:9-20.

44. Bacterial Endotoxins Test (general information 85). In: The United States pharmacopeia, 36th rev., and The national formulary, 31st ed. Rockville, MD: United States Pharmacopeial Convention; 2013.
45. Siegel SE, Nachum R, Leimbrock S, Karon M. Detection of bacterial endotoxin in antitumor agents. Cancer Treat Rep 1976;60:9-15.

46. Fumarola D. Possible endotoxin contamination in some antitumor agents preparations: study with the limulus amebocyte lysate test. Farm Ed Prat 1977;32:444-8.

47. Guide to good practices for the preparation of medicinal products in healthcare establishments PE 010-4. Pharmaceutical inspection cooperation scheme. PIC/S, March 2014. Available from: https:// www.gmp-compliance.org/guidemgr/files/PICS/PE-010-4-GUIDETO-GOOD-PRACTICES-1.PDF [Accessed Jan 2020]. 